\title{
MAXIMAL RATES OF DECAY OF SOLUTIONS OF PARTIAL DIFFERENTIAL EQUATIONS
}

\author{
BY WALTER LITTMAN
}

Communicated by M. H. Protter, May 23, 1969

It has been proved by C. Morawetz [2] that if $u(x, t)$ is a solution of the relativistic wave equation

$$
u_{t t}-\Delta u+u=0
$$

for all $x=\left(x_{1}, x_{2}, \cdots, x_{n}\right)$ and $t$, having finite energy at $t=0$, and vanishing in the forward light cone $|x|<t, t>0$, then it must vanish identically. On the other hand the author [1] has obtained a generalization of Rellich's Theorem (concerning decay of solutions of the reduced wave equation $\Delta u+u=0$ ) to a class of (not necessarily elliptic) equations with constant coefficients of arbitrary order. The present note is intended to announce a number of results which are natural generalizations of and improvements of both aforementioned results. Detailed proofs will appear elsewhere.

Let $P(\xi)=P\left(\xi_{1}, \xi_{2}, \cdots, \xi_{N}\right)$ be a polynomial with real coefficients. Throughout, we make the following assumptions:

1. The real solution set $S$ of $P(\xi)=0$ is nonempty.

2. Grad $P(\xi) \neq 0$ in $S$, and hence $S$ is a smooth $N-1$ dimensional manifold.

3. The Gaussian curvature of $S$ never vanishes.

Assign a unit normal $n$ to each point of $S$, varying continuously. The totality of all $n$ fill an open set $\pi$ on the unit sphere, giving rise to an open cone $\nVdash$ in $R^{N}$ in the sense that $\nVdash$ consists of all $r n, n \in \Re$, $r \geqq 0$.

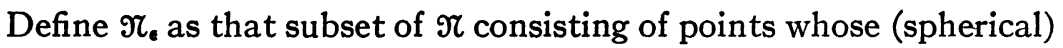
distance to the boundary of $\mathscr{N}$ exceeds $\epsilon$. $\mathcal{K}_{\boldsymbol{e}}$ will denote the cone generated by $\Re_{e .}-\Re$ will denote the set of vectors $-n$, with $n \in \Re$, and similarly for $-\mathfrak{K}$. $\mathfrak{K}^{\prime}$ denotes the complement of $\mathscr{N}$ on the unit sphere, and $\mathfrak{K}^{\prime}$ the corresponding cone. $\bar{K}$ denotes the closure of $K$.

We will write

$$
L u \equiv P\left(\frac{1}{i} \frac{\partial}{\partial x_{1}}, \cdots, \frac{1}{i} \frac{\partial}{\partial x_{N}}\right) u \equiv P\left(\frac{1}{i} \frac{\partial}{\partial x}\right) u .
$$

Theorem I. Suppose, under the foregoing Assumptions $1-3, u(x)$ is a function satisfying 


$$
\begin{array}{ll}
P\left(\frac{1}{i} \frac{\partial}{\partial x}\right) u=0 & \text { in } R^{N}, \\
u=O\left(1+|x|^{\alpha}\right) & \text { for some } \alpha, \\
u=o\left(|x|^{(1-N) / 2}\right) & \text { uniformly in every } \mathcal{K}_{\epsilon},
\end{array}
$$

then $u \equiv 0$ in $R^{N}$.

Assumption 4. Each (complex) irreducible factor $P_{j}$ has an $N-1$ dimensional real solution set $S_{j}$.

Theorem II. Under Assumptions 1-4, if

$$
\begin{array}{rlr}
L u & =f \text { has compact support, } \\
u & =O\left(1+|x|^{\alpha}\right) \quad \text { for some } \alpha, \text { uniformly in } R^{N}, \\
u & =o\left(|x|^{(1-N) / 2}\right) \quad \text { uniformly in every } K_{\varepsilon},-K_{\epsilon},
\end{array}
$$

then $u$ has compact support.

Note. For the equation $\Delta u+u=0$ the polynomial $P(\xi)=1-|\xi|^{2}$, and $S$ is the sphere $\xi_{1}^{2}+\xi_{2}^{2}+\cdots \xi_{N}^{2}=1$. $K^{2}, K_{\epsilon}$ are simply $R^{N}$, and we obtain Rellich's Theorem from Theorem II. On the other hand, for the equation $u_{t t}-\Delta u+u=0$, setting

$$
t=\xi_{N}, P(\xi)=\xi_{1}^{2}+\xi_{2}^{2}+\cdots+\xi_{N-1}^{2}-\xi_{N}^{2}+1,
$$

and $S$ is the two sheeted hyperboloid

$$
\xi_{1}^{2}+\xi_{2}^{2}+\xi_{N-1}^{2}-\xi_{N}^{2}+1=0 .
$$

If we assign $\boldsymbol{n}$ so that it always points "up", i.e., its $N$ th component is always positive, then $\mathscr{K}$ is the forward light cone, and applying Theorem I we obtain C. Morawetz's result, even a slight improvement. However, if we choose $\boldsymbol{n}$ to point "up" in the "upper" sheet and "down" in the "lower" sheet, $\mathfrak{K}$ will be the union of the forward and backward light cones, and we get a worse result. Thus $\boldsymbol{n}$ has to be chosen with care.

Assumption 5. (a) $P((1 / i)(\partial / \partial x))$ is hyperbolic with respect to $x_{N}$.

(b) $\overline{\mathcal{K}}$ does not intersect the set $x_{N} \leqq 0$ except at the origin.

TheOREM III. Under Assumptions 1-5, suppose

$L u=0$ for $x_{N}>0$,

the Cauchy data of $u$ has compact support on $x_{N}=0$,

$u\left(x_{1}, x_{2} \cdots x_{N-1}, 0\right)$ has compact support in $R^{N-1}$, $u=O\left(1+|x|^{\alpha}\right)$ uniformly for $x_{N}>0$ for some $\alpha$, 
$u=o\left(|x|^{(1-N) / 2}\right)$ uniformly in some open cone $\varkappa^{*}$ such that $\Re^{*} \cap \varkappa_{j}$ $\neq 0 j=1, \cdots, r$ (where each $\mathcal{K}_{j}$ corresponds to the surface $S_{j}$ and $r$ is the number of these surfaces).

Then $u=0$ for $x_{N}>0$.

Acknowledgment. The results described in this announcement were obtained in part under contract NONR 285(46) while the author was a visiting member at the Courant Institute (1957-58), and in part under Air Force contract AF-AFOSR-883-67 at the University of Minnesota.

\section{BIBLIOGRAPHY}

1. W. Littman, Decay at infinity of solutions to partial differential equations with constant coefficients, Trans. Amer. Math. Soc. 123 (1966), 449-459.

2. C. S. Morawetz, A uniqueness theorem for the relativistic wave equation, Comm. Pure Appl. Math. 16 (1963), 353-362.

University of Minnesota, Minneapolis, Minnesota 55455 